\title{
NEW UNIVALENCE CRITERIA FOR AN INTEGRAL OPERATOR WITH MOCANU'S AND ŞERB'S LEMMA
}

\author{
C. BĂRBAtu, D. Breaz
}

ABSTRACT. In this paper we consider an integral operator for analytic functions in the open unit disk $U$ and we obtain sufficient conditions for univalence of this integral operator, using Mocanu's and Şerb's Lemma.

2010 Mathematics Subject Classification: 30C45.

Keywords: Integral operator; univalence; unit disk.

\section{INTRODUCTION}

Let $\mathcal{A}$ be the class of the functions $f$ which are analytic in the open unit disk $\mathcal{U}=\{z \in \mathbb{C}:|z|<1\}$ and $f(0)=f^{\prime}(0)-1=0$.

We denote by $S$ the subclass of $A$ consisting of functions $f \in A$, which are univalent in $\mathcal{U}$.

We consider the integral operator

$$
\mathcal{T}_{n}(z)=\left\{\delta \int_{0}^{z} t^{\delta-1} \coprod_{i=1}^{n}\left[\left(\frac{f_{i}(t)}{t}\right)^{\alpha_{i}-1} \cdot\left(g_{i}^{\prime}(t)\right)^{\beta_{i}} \cdot\left(\frac{h_{i}(t)}{k_{i}(t)}\right)^{\gamma_{i}} \cdot\left(\frac{\left.h_{i}{ }^{\prime}(t)\right)}{k_{i}{ }^{\prime}(t)}\right)^{\delta_{i}}\right] \mathrm{dt}\right\}^{\frac{1}{\delta}}
$$

for $f_{i}, g_{i}, h_{i}, k_{i} \in \mathcal{A}$ and the complex numbers $\delta, \alpha_{i}, \beta_{i}, \gamma_{i}, \delta_{i}$, with $\delta \neq 0, i=\overline{1, n}$, $n \in \mathbb{N} \backslash\{0\}$. 


\section{Preliminary Results}

In order to prove main results we will use the following lemmas.

Lemma 1. [7] Let $\gamma, \delta$ be complex numbers, Re $>0$ and $f \in \mathcal{A}$. If

$$
\frac{1-|z|^{2 \operatorname{Re} \gamma}}{\operatorname{Re\gamma }}\left|\frac{\left.z f^{\prime \prime}(z)\right)}{f^{\prime}(z)}\right| \leq 1,
$$

for all $z \in \mathcal{U}$, then for any complex number $\delta, R e \delta \geq \operatorname{Re} \gamma$, the function $F_{\delta}$ defined by

$$
F_{\delta}(z)=\left(\delta \int_{0}^{z} t^{\delta-1} f^{\prime}(t) d t\right)^{\frac{1}{\delta}},
$$

is regular and univalent in $\mathcal{U}$.

Lemma 2. [5] Let $M_{0}=1,5936$... the positive solution of equation

$$
(2-M) e^{M}=2 .
$$

If $f \in \mathcal{A}$ and

$$
\left|\frac{\left.f^{\prime \prime}(z)\right)}{f^{\prime}(z)}\right| \leq M_{0}
$$

for $z \in \mathcal{U}$, then

$$
\left|\frac{\left.z f^{\prime}(z)\right)}{f(z)}-1\right| \leq 1, \quad(z \in U)
$$

The edge $M_{0}$ is sharp.

Lemma 3. [3] Let $f$ be the function regular in the disk $\mathcal{U}_{R}=\{z \in \mathbb{C}:|z|<R\}$ with $|f(z)|<M$, M fixed. If $f(z)$ has in $z=0$ one zero with multiply $\geq m$, then

$$
|f(z)| \leq \frac{M}{R^{m}} z^{m}
$$

the equality for $z \neq 0$ can hold only if

$$
f(z)=e^{i \theta} \frac{M}{R^{m}} z^{m}
$$

where $\theta$ is constant. 
C. Bărbatu, D. Breaz - New Univalence Criteria for an Integral Operator ...

\section{MAin Results}

Theorem 4. Let $\gamma, \delta, \alpha_{i}, \beta_{i}, \gamma_{i}, \delta_{i}$ be complex numbers, $c=$ Re $\gamma>0, M_{0}$ the positive solution of the equation (2), $M_{0}=1,5936 \ldots$ and $f_{i}, g_{i}, h_{i}, k_{i} \in \mathcal{A}, f_{i}(z)=z+$ $a_{2 i} z^{2}+a_{3 i} z^{3}+\ldots, g_{i}(z)=z+b_{2 i} z^{2}+b_{3 i} z^{3}+\ldots ., h_{i}(z)=z+c_{2 i} z^{2}+c_{3 i} z^{3}+\ldots$. $k_{i}(z)=z+d_{2 i} z^{2}+d_{3 i} z^{3}+\ldots, i=\overline{1, n}$ If

$$
\left|\frac{f_{i}^{\prime \prime}(z)}{f_{i}^{\prime}(z)}\right| \leq M_{0}, \quad\left|\frac{g_{i}^{\prime \prime}(z)}{g_{i}^{\prime}(z)}\right| \leq M_{0}, \quad\left|\frac{h_{i}^{\prime \prime}(z)}{h_{i}^{\prime}(z)}\right| \leq M_{0}, \quad\left|\frac{k_{i}^{\prime \prime}(z)}{k_{i}^{\prime}(z)}\right| \leq M_{0}
$$

for all $z \in \mathcal{U}, i=\overline{1, n}$ and

$$
\frac{1}{c} \sum_{i=1}^{n}\left|\alpha_{i}-1\right|+\frac{2 M_{0}}{(2 c+1)^{\frac{2 c+1}{2 c}}} \sum_{i=1}^{n}\left|\beta_{i}\right|+\frac{2}{c} \sum_{i=1}^{n}\left|\gamma_{i}\right|+\frac{4 M_{0}}{(2 c+1)^{\frac{2 c+1}{2 c}}} \sum_{i=1}^{n}\left|\delta_{i}\right| \leq 1,
$$

then for all $\delta$ complex numbers, Re $\delta \geq$ Re $\gamma$, the integral operator $\mathcal{T}_{n}$, given by (1) is in the class $\mathcal{S}$.

Proof. Let us define the function

$$
H_{n}(z)=\int_{0}^{z} \prod_{i=1}^{n}\left[\left(\frac{f_{i}(t)}{t}\right)^{\alpha_{i}-1} \cdot\left(g_{i}^{\prime}(t)\right)^{\beta_{i}} \cdot\left(\frac{h_{i}(t)}{k_{i}(t)}\right)^{\gamma_{i}} \cdot\left(\frac{h_{i}{ }^{\prime}(t)}{k_{i}{ }^{\prime}(t)}\right)^{\delta_{i}}\right] \mathrm{dt},
$$

for $f_{i}, g_{i}, h_{i}, k_{i} \in \mathcal{A}, i=\overline{1, n}$.

The function $H_{n}$ is regular in $\mathcal{U}$ and satisfy the following usual normalization conditions $H_{n}(0)=H_{n}^{\prime}(0)-1=0$.

We have

$$
\begin{aligned}
& \frac{z H_{n}^{\prime \prime}(z)}{H_{n}^{\prime}(z)}=\sum_{i=1}^{n}\left[\left(\alpha_{i}-1\right)\left(\frac{z f_{i}^{\prime}(z)}{f_{i}(z)}-1\right)+\beta_{i} \frac{z g_{i}^{\prime \prime}(z)}{g_{i}^{\prime}(z)}\right]+ \\
& +\sum_{i=1}^{n}\left[\gamma_{i}\left(\frac{z h_{i}^{\prime}(z)}{h_{i}(z)}-\frac{z k_{i}^{\prime}(z)}{k_{i}(z)}\right)+\delta_{i}\left(\frac{z h_{i}^{\prime \prime}(z)}{h_{i}^{\prime}(z)}-\frac{z k_{i}^{\prime \prime}(z)}{k_{i}^{\prime}(z)}\right)\right],
\end{aligned}
$$

for all $z \in \mathcal{U}$.

Therefore

$$
\frac{1-|z|^{2 c}}{c}\left|\frac{z H_{n}^{\prime \prime}(z)}{H_{n}^{\prime}(z)}\right| \leq \frac{1-|z|^{2 c}}{c} \sum_{i=1}^{n}\left[\left|\alpha_{i}-1\right|\left|\frac{z f_{i}^{\prime}(z)}{f_{i}(z)}-1\right|+\left|\beta_{i}\right|\left|\frac{z g_{i}^{\prime \prime}(z)}{g_{i}^{\prime}(z)}\right|\right]+
$$


C. Bărbatu, D. Breaz - New Univalence Criteria for an Integral Operator ...

$$
+\left[\left|\gamma_{i}\right|\left(\left|\frac{z h_{i}^{\prime}(z)}{h_{i}(z)}-1\right|+\left|\frac{z k_{i}^{\prime}(z)}{k_{i}(z)}-1\right|\right)+\left|\delta_{i}\right|\left(\left|\frac{z h_{i}^{\prime \prime}(z)}{h_{i}^{\prime}(z)}\right|+\left|\frac{z k_{i}^{\prime \prime}(z)}{k_{i}^{\prime}(z)}\right|\right)\right],
$$

for all $z \in \mathcal{U}$.

Using (3), (4) and Lemma Mocanu and Şerb, from (5) we get

$$
\left|\frac{z f_{i}^{\prime}(z)}{f_{i}(z)}-1\right|<1, \quad\left|\frac{z h_{i}^{\prime}(z)}{h_{i}(z)}-1\right|<1, \quad\left|\frac{z k_{i}^{\prime}(z)}{k_{i}(z)}-1\right|<1,
$$

for all $z \in \mathcal{U}, i=\overline{1, n}$ and hence, we have

$$
\begin{gathered}
\frac{1-|z|^{2 c}}{c}\left|\frac{z H_{n}^{\prime \prime}(z)}{H_{n}^{\prime}(z)}\right| \leq \frac{1-|z|^{2 c}}{c} \sum_{i=1}^{n}\left|\alpha_{i}-1\right|+ \\
+\frac{1-|z|^{2 c}}{c}|z| M_{0} \sum_{i=1}^{n}\left|\beta_{i}\right|+\frac{1-|z|^{2 c}}{c} 2 \sum_{i=1}^{n}\left|\gamma_{i}\right|+\frac{1-|z|^{2 c}}{c}|z| 2 M_{0} \sum_{i=1}^{n}\left|\delta_{i}\right|,
\end{gathered}
$$

for all $z \in \mathcal{U}$

Since

$$
\max _{|z| \leq 1} \frac{\left(1-|z|^{2 c}\right)|z|}{c}=\frac{2}{(2 c+1)^{\frac{2 c+1}{2 c}}},
$$

from (6) and (7) we obtain

$$
\begin{gathered}
\frac{1-|z|^{2 c}}{c}\left|\frac{z H_{n}^{\prime \prime}(z)}{H_{n}^{\prime}(z)}\right| \leq \\
\frac{1}{c} \sum_{i=1}^{n}\left|\alpha_{i}-1\right|+\frac{2 M_{0}}{(2 c+1)^{\frac{2 c+1}{2 c}}} \sum_{i=1}^{n}\left|\beta_{i}\right|+\frac{2}{c} \sum_{i=1}^{n}\left|\gamma_{i}\right| \frac{4 M_{0}}{(2 c+1)^{\frac{2 c+1}{2 c}}} \sum_{i=1}^{n}\left|\delta_{i}\right|,
\end{gathered}
$$

for all $z \in \mathcal{U}, i=\overline{1, n}$.

Using(6), from (8) we have

$$
\frac{1-|z|^{2 c}}{c}\left|\frac{z H_{n}^{\prime \prime}(z)}{H_{n}^{\prime}(z)}\right| \leq 1
$$

Now, from (9), by Lemma 2.1, it results that the integral operator $\mathcal{T}_{n}$, given by $(1)$ is in the class $\mathcal{S}$. 
C. Bărbatu, D. Breaz - New Univalence Criteria for an Integral Operator ...

Letting $\delta=1$ in Theorem 3.1, we have

Corollary 5. Let $\gamma, \alpha_{i}, \beta_{i}, \gamma_{i}, \delta_{i}$ be complex numbers, $0<$ Re $\gamma \leq 1, c=$ Re $\gamma$, $M_{0}$ the positive solution of the equation (3), $M_{0}=1,5936 \ldots$ and $f_{i}, g_{i}, h_{i}, k_{i} \in \mathcal{A}$, $f_{i}(z)=z+a_{2 i} z^{2}+a_{3 i} z^{3}+\ldots ., g_{i}(z)=z+b_{2 i} z^{2}+b_{3 i} z^{3}+\ldots ., h_{i}(z)=z+c_{2 i} z^{2}+c_{3 i} z^{3}+\ldots$. $k_{i}(z)=z+d_{2 i} z^{2}+d_{3 i} z^{3}+\ldots, i=\overline{1, n}$.

If

$$
\left|\frac{f_{i}^{\prime \prime}(z)}{f_{i}^{\prime}(z)}\right| \leq M_{0}, \quad\left|\frac{g_{i}^{\prime \prime}(z)}{g_{i}^{\prime}(z)}\right| \leq M_{0}, \quad\left|\frac{h_{i}^{\prime \prime}(z)}{h_{i}^{\prime}(z)}\right| \leq M_{0}, \quad\left|\frac{k_{i}^{\prime \prime}(z)}{k_{i}^{\prime}(z)}\right| \leq M_{0},
$$

for all $z \in \mathcal{U}, i=\overline{1, n}$ and

$$
\frac{1}{c} \sum_{i=1}^{n}\left|\alpha_{i}-1\right|+\frac{2 M_{0}}{(2 c+1)^{\frac{2 c+1}{2 c}}} \sum_{i=1}^{n}\left|\beta_{i}\right|+\frac{2}{c} \sum_{i=1}^{n}\left|\gamma_{i}\right|+\frac{4 M_{0}}{(2 c+1)^{\frac{2 c+1}{2 c}}} \sum_{i=1}^{n}\left|\delta_{i}\right| \leq 1,
$$

then the integral operator $\mathcal{F}_{n}$ defined by

$$
\mathcal{F}_{n}(z)=\int_{0}^{z} \coprod_{i=1}^{n}\left[\left(\frac{f_{i}(t)}{t}\right)^{\alpha_{i}-1} \cdot\left(g_{i}^{\prime}(t)\right)^{\beta_{i}} \cdot\left(\frac{h_{i}(t)}{k_{i}(t)}\right)^{\gamma_{i}} \cdot\left(\frac{\left.h_{i}{ }^{\prime}(t)\right)}{k_{i}{ }^{\prime}(t)}\right)^{\delta_{i}}\right] d t,
$$

is in the class $\mathcal{S}$.

Letting $\delta=1$ and $\delta_{1}=\delta_{2}=\ldots=\delta_{n}=0$ in Theorem 3.1, we have

Corollary 6. Let $\gamma, \alpha_{i}, \beta_{i}, \gamma_{i}$ be complex numbers, $0<$ Re $\gamma \leq 1, c=R e \gamma, M_{0}$ the positive solution of the equation (2), $M_{0}=1,5936 \ldots$ and $f_{i}, g_{i}, h_{i}, k_{i} \in \mathcal{A}, f_{i}(z)=$ $z+a_{2 i} z^{2}+a_{3 i} z^{3}+\ldots ., g_{i}(z)=z+b_{2 i} z^{2}+b_{3 i} z^{3}+\ldots ., h_{i}(z)=z+c_{2 i} z^{2}+c_{3 i} z^{3}+\ldots$. $k_{i}(z)=z+d_{2 i} z^{2}+d_{3 i} z^{3}+\ldots ., i=\overline{1, n}$.

If

$$
\left|\frac{f_{i}^{\prime \prime}(z)}{f_{i}^{\prime}(z)}\right| \leq M_{0}, \quad\left|\frac{g_{i}^{\prime \prime}(z)}{g_{i}^{\prime}(z)}\right| \leq M_{0}, \quad\left|\frac{h_{i}^{\prime \prime}(z)}{h_{i}^{\prime}(z)}\right| \leq M_{0}, \quad\left|\frac{k_{i}^{\prime \prime}(z)}{k_{i}^{\prime}(z)}\right| \leq M_{0}
$$

for all $z \in \mathcal{U}, i=\overline{1, n}$ and

$$
\frac{1}{c} \sum_{i=1}^{n}\left|\alpha_{i}-1\right|+\frac{2 M_{0}}{(2 c+1)^{\frac{2 c+1}{2 c}}} \sum_{i=1}^{n}\left|\beta_{i}\right|+\frac{2}{c} \sum_{i=1}^{n}\left|\gamma_{i}\right| \leq 1,
$$

then the integral operator $\mathcal{S}_{n}$ defined by

$$
\mathcal{S}_{n}(z)=\int_{0}^{z} \coprod_{i=1}^{n}\left[\left(\frac{f_{i}(t)}{t}\right)^{\alpha_{i}-1} \cdot\left(g_{i}^{\prime}(t)\right)^{\beta_{i}} \cdot\left(\frac{h_{i}(t)}{k_{i}(t)}\right)^{\gamma_{i}}\right] d t
$$

is in the class $\mathcal{S}$. 
C. Bărbatu, D. Breaz - New Univalence Criteria for an Integral Operator ...

Letting $\delta=1$ and $\beta_{1}=\beta_{2}=\ldots=\beta_{n}=0$ in Theorem 3.1, we obtain

Corollary 7. Let $\gamma, \alpha_{i}, \gamma_{i}, \delta_{i}$ be complex numbers, $0<\operatorname{Re} \gamma \leq 1, c=\operatorname{Re} \gamma, i=\overline{1, n}$, $M_{0}$ the positive solution of the equation (2), $M_{0}=1,5936 \ldots$ and $f_{i}, h_{i}, k_{i} \in \mathcal{A}$, $f_{i}(z)=z+a_{2 i} z^{2}+a_{3 i} z^{3}+\ldots ., h_{i}(z)=z+c_{2 i} z^{2}+c_{3 i} z^{3}+\ldots ., k_{i}(z)=z+d_{2 i} z^{2}+$ $d_{3 i} z^{3}+\ldots, i=\overline{1, n}$.

If

$$
\left|\frac{f_{i}^{\prime \prime}(z)}{f_{i}^{\prime}(z)}\right| \leq M_{0}, \quad\left|\frac{h_{i}^{\prime \prime}(z)}{h_{i}^{\prime}(z)}\right| \leq M_{0}, \quad\left|\frac{k_{i}^{\prime \prime}(z)}{k_{i}^{\prime}(z)}\right| \leq M_{0},
$$

for all $z \in \mathcal{U}, i=\overline{1, n}$ and

$$
\frac{1}{c} \sum_{i=1}^{n}\left|\alpha_{i}-1\right|+\frac{2}{c} \sum_{i=1}^{n}\left|\gamma_{i}\right|+\frac{4 M_{0}}{(2 c+1)^{\frac{2 c+1}{2 c}}} \sum_{i=1}^{n}\left|\delta_{i}\right| \leq 1,
$$

then the integral operator $\mathcal{X}_{n}$ defined by

$$
\mathcal{X}_{n}(z)=\int_{0}^{z} \coprod_{i=1}^{n}\left[\left(\frac{f_{i}(t)}{t}\right)^{\alpha_{i}-1} \cdot\left(\frac{h_{i}(t)}{k_{i}(t)}\right)^{\gamma_{i}} \cdot\left(\frac{\left.h_{i}{ }^{\prime}(t)\right)}{k_{i}{ }^{\prime}(t)}\right)^{\delta_{i}}\right] d t
$$

is in the class $\mathcal{S}$.

Letting $\delta=1$ and $\alpha_{1}=\alpha_{2}=\ldots=\alpha_{n}=0$ in Theorem 3.1, we have

Corollary 8. Let $\gamma, \beta_{i}, \gamma_{i}, \delta_{i}$ be complex numbers, $0<\operatorname{Re} \gamma \leq 1, c=\operatorname{Re} \gamma, M_{0}$ the positive solution of the equation (2), $M_{0}=1,5936 \ldots$ and $g_{i}, h_{i}, k_{i} \in \mathcal{A}, g_{i}(z)=$ $z+b_{2 i} z^{2}+b_{3 i} z^{3}+\ldots, h_{i}(z)=z+c_{2 i} z^{2}+c_{3 i} z^{3}+\ldots, k_{i}(z)=z+d_{2 i} z^{2}+d_{3 i} z^{3}+\ldots$. $i=\overline{1, n}$.

\section{If}

$$
\left|\frac{g_{i}^{\prime \prime}(z)}{g_{i}^{\prime}(z)}\right| \leq M_{0}, \quad\left|\frac{h_{i}^{\prime \prime}(z)}{h_{i}^{\prime}(z)}\right| \leq M_{0}, \quad\left|\frac{k_{i}^{\prime \prime}(z)}{k_{i}^{\prime}(z)}\right| \leq M_{0},
$$

for all $z \in \mathcal{U}, i=\overline{1, n}$ and

$$
\frac{2 M_{0}}{(2 c+1)^{\frac{2 c+1}{2 c}}} \sum_{i=1}^{n}\left|\beta_{i}\right|+\frac{2}{c} \sum_{i=1}^{n}\left|\gamma_{i}\right|+\frac{4 M_{0}}{(2 c+1)^{\frac{2 c+1}{2 c}}} \sum_{i=1}^{n}\left|\delta_{i}\right| \leq 1,
$$

then the integral operator $\mathcal{D}_{n}$ defined by

$$
\mathcal{D}_{n}(z)=\int_{0}^{z} \coprod_{i=1}^{n}\left[\left(g_{i}^{\prime}(t)\right)^{\beta_{i}} \cdot\left(\frac{h_{i}(t)}{k_{i}(t)}\right)^{\gamma_{i}} \cdot\left(\frac{\left.h_{i}{ }^{\prime}(t)\right)}{k_{i}{ }^{\prime}(t)}\right)^{\delta_{i}}\right] d t,
$$

is in the class $\mathcal{S}$. 
C. Bărbatu, D. Breaz - New Univalence Criteria for an Integral Operator ...

Letting $\delta=1$ and $\gamma_{1}=\gamma_{2}=\ldots=\gamma_{n}=0$ in Theorem 3.1, we have

Corollary 9. Let $\gamma, \alpha_{i}, \beta_{i}, \delta_{i}$ be complex numbers, $0<\operatorname{Re} \gamma \leq 1, c=\operatorname{Re} \gamma, M_{0}$ the positive solution of the equation (2), $M_{0}=1,5936 \ldots$ and $f_{i}, g_{i}, h_{i}, k_{i} \in \mathcal{A}, f_{i}(z)=$ $z+a_{2 i} z^{2}+a_{3 i} z^{3}+\ldots ., g_{i}(z)=z+b_{2 i} z^{2}+b_{3 i} z^{3}+\ldots ., h_{i}(z)=z+c_{2 i} z^{2}+c_{3 i} z^{3}+\ldots .$, $k_{i}(z)=z+d_{2 i} z^{2}+d_{3 i} z^{3}+\ldots ., i=\overline{1, n}$.

If

$$
\left|\frac{f_{i}^{\prime \prime}(z)}{f_{i}^{\prime}(z)}\right| \leq M_{0}, \quad\left|\frac{g_{i}^{\prime \prime}(z)}{g_{i}^{\prime}(z)}\right| \leq M_{0}, \quad\left|\frac{h_{i}^{\prime \prime}(z)}{h_{i}^{\prime}(z)}\right| \leq M_{0}, \quad\left|\frac{k_{i}^{\prime \prime}(z)}{k_{i}^{\prime}(z)}\right| \leq M_{0},
$$

for all $z \in \mathcal{U}, i=\overline{1, n}$ and

$$
\frac{1}{c} \sum_{i=1}^{n}\left|\alpha_{i}-1\right|+\frac{2 M_{0}}{(2 c+1)^{\frac{2 c+1}{2 c}}} \sum_{i=1}^{n}\left|\beta_{i}\right|+\frac{4 M_{0}}{(2 c+1)^{\frac{2 c+1}{2 c}}} \sum_{i=1}^{n}\left|\delta_{i}\right| \leq 1,
$$

then the integral operator $\mathcal{Y}_{n}$ defined by

$$
\mathcal{Y}_{n}(z)=\int_{0}^{z} \coprod_{i=1}^{n}\left[\left(\frac{f_{i}(t)}{t}\right)^{\alpha_{i}-1} \cdot\left(g_{i}^{\prime}(t)\right)^{\beta_{i}} \cdot\left(\frac{\left.h_{i}{ }^{\prime}(t)\right)}{k_{i}{ }^{\prime}(t)}\right)^{\delta_{i}}\right] d t,
$$

is in the class $\mathcal{S}$.

Letting $n=1, \delta=\gamma=\alpha$ and $\alpha_{i}-1=\beta_{i}=\gamma_{i}$ in Theorem 3.1, we obtain

Corollary 10. Let $\alpha$ be complex number, $a=$ Re $\alpha>0, M_{0}$ the positive solution of the equation (2), $M_{0}=1,5936 \ldots$ and $f, g, h, k \in \mathcal{A}, f(z)=z+a_{2} z^{2}+a_{3} z^{3}+\ldots$, $g(z)=z+b_{2} z^{2}+b_{3} z^{3}+\ldots ., h(z)=z+c_{2} z^{2}+c_{3} z^{3}+\ldots ., k(z)=z+d_{2} z^{2}+d_{3} z^{3}+\ldots .$.

If

$$
\left|\frac{f^{\prime \prime}(z)}{f^{\prime}(z)}\right| \leq M_{0}, \quad\left|\frac{g^{\prime \prime}(z)}{g^{\prime}(z)}\right| \leq M_{0}, \quad\left|\frac{h^{\prime \prime}(z)}{h^{\prime}(z)}\right| \leq M_{0}, \quad\left|\frac{k^{\prime \prime}(z)}{k^{\prime}(z)}\right| \leq M_{0}
$$

for all $z \in \mathcal{U}$, and

$$
\frac{\alpha-1}{a}+\frac{2 \beta M_{0}}{(2 a+1)^{\frac{2 a+1}{2 a}}}+\frac{2 \gamma}{a}+\frac{4 \delta M_{0}}{(2 a+1)^{\frac{2 a+1}{2 a}}} \leq 1,
$$

then the integral operator $\mathcal{T}$ defined by

$$
\mathcal{T}(z)=\left[\alpha \int_{0}^{z} t^{\alpha-1}\left(f(t) \cdot g^{\prime}(t) \cdot \frac{h(t)}{k(t)} \cdot \frac{\left.h^{\prime}(t)\right)}{k^{\prime}(t)}\right)^{\alpha-1} d t\right]^{\frac{1}{\alpha}},
$$

is in the class $\mathcal{S}$. 
C. Bărbatu, D. Breaz - New Univalence Criteria for an Integral Operator ...

Letting $M_{0}=M$ from (3) in Theorem 3.1, we obtain

Corollary 11. Let $\gamma, \delta, \alpha_{i}, \beta_{i}, \gamma_{i}, \delta_{i}$ be complex numbers, $c=$ Re $\gamma>0, M$ a positive number and $M_{0}$ the positive solution of the equation (2), $M_{0}=1,5936 \ldots$ and $f_{i}, g_{i}, h_{i}, k_{i} \in \mathcal{A}, f_{i}(z)=z+a_{2 i} z^{2}+a_{3 i} z^{3}+\ldots, g_{i}(z)=z+b_{2 i} z^{2}+b_{3 i} z^{3}+\ldots$. $h_{i}(z)=z+c_{2 i} z^{2}+c_{3 i} z^{3}+\ldots, k_{i}(z)=z+d_{2 i} z^{2}+d_{3 i} z^{3}+\ldots ., i=\overline{1, n}$

If

$$
\left|\frac{f_{i}^{\prime \prime}(z)}{f_{i}^{\prime}(z)}\right| \leq M_{0}, \quad\left|\frac{g_{i}^{\prime \prime}(z)}{g_{i}^{\prime}(z)}\right| \leq M, \quad\left|\frac{h_{i}^{\prime \prime}(z)}{h_{i}^{\prime}(z)}\right| \leq M_{0}, \quad\left|\frac{k_{i}^{\prime \prime}(z)}{k_{i}^{\prime}(z)}\right| \leq M_{0},
$$

for all $z \in \mathcal{U}, i=\overline{1, n}$ and

$$
\frac{1}{c} \sum_{i=1}^{n}\left|\alpha_{i}-1\right|+\frac{2 M}{c} \sum_{i=1}^{n}\left|\beta_{i}\right|+\frac{2}{c} \sum_{i=1}^{n}\left|\gamma_{i}\right|+\frac{4 M_{0}}{(2 c+1)^{\frac{2 c+1}{2 c}}} \sum_{i=1}^{n}\left|\delta_{i}\right| \leq 1,
$$

then $f_{i}, g_{i}, h_{i}, k_{i} \in \mathcal{S}, i=\overline{1, n}$ and for all $\delta$ complex numbers, Re $\delta \geq$ Re $\gamma$, the integral operator $\mathcal{T}_{n}$, given by (1) is in the class $\mathcal{S}$.

\section{REFERENCES}

[1] D. Breaz, N. Breaz, Two Integral Operators. Studia Univ."Babes-Bolyai", ClujNapoca, Mathematica, 47(2002), no. 3, pg. 13-21.

[2] D. Breaz, N. Breaz, H. M. Srivastava, An extension of the univalent condition for a family of integral operators. Appl. Math. Lett., 22(2009), no. 3, 41-44.

[3] O. Mayer, The Functions Theory of the One Variable Complex . Acad. Ed., Bucuresti, Romania, 1981, 101-117.

[4] P. T. Mocanu, T. Bulboaca, G. S. Salagean, Teoria geometrica a functiilor univalente. Casa Cartii de Stiinta, Cluj Napoca (1999), 77-81.

[5] P. T. Mocanu, I. Şerb, A sharp simple criterion for a subclass of starlike functions. Complex variables, 32(1997), 161-168.

[6] S. Ozaki, M. Nunokawa, The Schwarzian derivative and univalent functions. Proceedings of the American Mathematical Society, Mathematics, 33(1972), 392-394.

[7] N. N. Pascu, An a univalence criterion II. Itinerant Seminar on Functional Equations, Approximation and Convexity, Cluj Napoca 1985, 153-154.

[8] V. Pescar, New univalence criteria for some integral operators. Studia Univ."Babes-Bolyai", Cluj-Napoca, Mathematica, 59(2014), no. 2, 185-192. 
C. Bărbatu, D. Breaz - New Univalence Criteria for an Integral Operator ...

[9] V. Pescar, N. Breaz, Mocanu and Şerb type univalence criteria for some general integral operators. Acta Universitatis Apulensis, 44(2015), 1-8.

Camelia Bărbatu

Faculty of Mathematics and Computer Science, "Babeş-Bolyai" University,

Cluj Napoca, Romania

email: camipode@yahoo.com

Daniel Breaz

Department of Mathematics, Faculty of Exact Sciences and Engineering,

"1 Decembrie" University,

Alba Iulia, Romania

email: dbreaz@uab.ro 